\title{
Recognition of Elementary Arm Movements Using Orientation of a Tri-axial Accelerometer Located Near the Wrist
}

\author{
Dwaipayan Biswas ${ }^{1}$, Daniele Corda ${ }^{2}$, Giovanni Baldus ${ }^{2}$, Andy Cranny ${ }^{1}$, Koushik Maharatna ${ }^{1}$, \\ Josy Achner ${ }^{3}$, Jasmin Klemke ${ }^{3}$, Michael Jöbges ${ }^{3}$ and Steffen Ortmann ${ }^{4}$
}

\author{
${ }^{1}$ Faculty of Physical Sciences and Engineering, University of Southampton, Hampshire, UK \\ ${ }^{2}$ National Research Council of Italy (CNR), Institute of Clinical Physiology (IFC), Pisa, Italy \\ ${ }^{3}$ Brandenburg Klinik, Bernau, Berlin, Germany \\ ${ }^{4}$ IHP, Leibniz-Institute for Innovative Microelectronics, Frankfurt (Oder), Germany \\ Email: $\underline{\text { db9g10@ecs.soton.ac.uk }}$
}

\begin{abstract}
In this paper we present a method for recognising three fundamental movements of the human arm (reach and retrieve, lift cup to mouth, rotation of the arm) by determining the orientation of a tri-axial accelerometer located near the wrist. Our objective is to detect the occurrence of such movements performed with the impaired arm of a stroke patient during normal daily activities as a means to assess their rehabilitation. The method relies on accurately mapping transitions of predefined, standard orientations of the accelerometer to corresponding elementary arm movements. To evaluate the technique, kinematic data was collected from four healthy subjects and four stroke patients as they performed a number of activities involved in a representative activity of daily living, 'making-a-cup-of-tea'. Our experimental results show that the proposed method can independently recognise all three of the elementary upper limb movements investigated with accuracies in the range $91 \%-99 \%$ for healthy subjects and $70 \%-85 \%$ for stroke patients.
\end{abstract}

Keywords: Accelerometer, activity recognition, movement classification, remote health monitoring, wireless body area network.

\section{Introduction}

Motion tracking of the human body has been applied in a wide range of application areas such as remote health monitoring [1]-[3], human computer interaction [4], [5], sports coaching and training [6], [7], etc., using different sensing technologies including mechanical tracking, optical systems, acoustic tracking, radar systems and inertial/magnetic systems. The information extracted from such sensors and systems have been used to estimate limb orientation, compute limb joint angles, and in the development of kinematic models of the human body. In turn, this has helped in the qualification and quantification of physical activities [8]-[17]. For example, studies using kinematic data collected from stroke patients have been undertaken to determine whether a quantitative electronic scoring system can be used to augment, or possibly replace, traditional appraisal methods performed by physiotherapists in assessing patient rehabilitation progress. The latter typically involves the clinician observing and grading movement quality of the upper limb during the performance of particular motor activities; a process that can be considered rather subjective. For example, Patel et al. used accelerometers located on the arm and chest to assess stroke patients as they performed a subset of the motor tasks pertaining to the Functional Ability Scale [18], whilst Subramanian et al. used kinematic data collected using an optical system with markers placed on the hand, elbow and shoulders of stroke patients, and compared this to scores obtained by the Fugl-Meyer Assessment method [19]. Both of these exemplar studies concluded 
that analysis of kinematic data was a valid method of appraising upper limb movement quality and demonstrated high correlation with the traditional, observational methods.

Advances in wireless sensor networks and Information and Communication Technology have played a key role in the miniaturization of low-cost, body-worn, inertial sensors (accelerometers and gyroscopes) that make possible cost efficient patient monitoring and rehabilitation within the home environment [20]-[22]. This is particularly relevant when considering the increasing healthcare costs of an ageing population, especially those related to the treatment of chronic arthritis, cardiovascular diseases or neurodegenerative diseases, and the desire to reduce the amount of time the patient spends at the clinic. Research and development in to Wireless Body Area Networks (WBANs), wherein a patient wears a number of sensors either directly on their body or contained within clothing or other facilitators [23], have enabled user data to be captured in a ubiquitous and continuous manner within natural environments. In such wearable systems, the data analysis primarily needs to be carried out at the sensor node yielding energy efficient solutions as compared to conventional remote monitoring approaches based on continuous transmission of vital sign data [3]. Hence, from the long-term system operation perspective, when implementing a WBAN that is comprised of heterogeneous sensors capturing physiological data, it is imperative to select data analysis algorithms that are computationally of low complexity. This is because energy consumption is directly proportional to the computational complexity of the processing algorithms used, and therefore higher complexity algorithms (although they may be more accurate) will drain the power source faster, limiting the scope of long-term continuous monitoring.

In this paper we describe a simple method to recognise three fundamental movements of the upper limb that are generally associated with activities of daily living (ADL), using data collected from a wrist-worn, wireless tri-axial accelerometer. The detection and classification of limb movements is beneficial in several remote health monitoring applications. For example, in many neurodegenerative pathologies regular exercise of the impaired limb is necessary to achieve rehabilitation. A system capable of detecting the use of the impaired limb (during prescribed exercise and during ADL) and classifying the type of movements performed would therefore be of use. Here, we focus on detecting specific upper limb movements of stroke patients, with varying degrees of motor impairment. Enumerating occurrences of these movements over time can act as an indicator of rehabilitation progress since the patient is more likely to repeat these movements as their motor function improves. The three movements investigated, along with examples of their daily occurrence, were: extension/flexion of the forearm (reach and retrieve object); rotation of the forearm about the elbow (drinking action); and rotation of the arm about the median axis (opening a door, using a key or pouring action). Our aim was to investigate a methodology that allows simple detection of these three movements with maximal accuracy, while accounting for their temporal and inter-subject variability, using a low-complexity algorithm. The purpose of this is to produce simple software code that can be readily translated into hardware in the form of a custom chip with low energy (power) requirement, which would help in the realisation of WBAN systems for home based telemedicine applications. The algorithm developed mines the accelerometer data searching for occurrences of 6 predefined reference orientations (or states) of the sensor. By analysing transitions between occurrences of these states, it is possible to predict the corresponding arm movements.

In this investigation experiments were performed with healthy subjects and with stroke patients with impaired upper limb mobility. Specifically, accelerometer data was collected as participants simulated the processes involved in a typical ADL: the act of 'making-a-cup-of-tea'. We used stroke patient data as an application scenario of telerehabilitation and also because of the larger variability it is likely to produce in the data, thus providing a means to test the robustness of our methodology. Our results show that the proposed method can recognise the three movements reasonably well across all the subjects using only a tri-axial accelerometer placed near the wrist.

The remainder of this paper is organized as follows. Section II presents a brief background study on human activity 
recognition with emphasis on the use of inertial sensors and identifies the motivation for this research. The experimental protocol is discussed in Section III whilst Section IV describes the data processing and the proposed algorithm. The analysis of the experimental results is provided in Section V and conclusions are drawn in Section VI.

\section{Background}

Human activity recognition in natural settings is an active research area that has been applied widely in the field of chronic disease management and rehabilitation [15]-[17]. It is also motivated by a wide range of mobile and ubiquitous computing applications which include personalisation of user interfaces [10], further aided by the development of inertial sensors. Radio-frequency identification (RFID) has also been used to monitor the movement of objects within the home environment that are typically encountered during daily living [24]. Another approach being used is fusing data from vision systems and inertial sensors to complement each other. This approach is, however, mainly restricted to indoor activities within a defined region under the un-hindered surveillance of the vision system. Furthermore, the use of high complexity image processing algorithms can result in slower analysis which can be particularly challenging if real-time information analysis is required [15].

In principle there are three steps for activity recognition using inertial sensors: 1) data capture by appropriate sensor; 2) segmentation of the captured data to identify the beginning and end of an activity; and 3) recognition of the activity using appropriate classification techniques. It may be noted that although the final two steps are in practise interrelated; individually they pose significant research challenges owing to the possible qualitative non-uniqueness of an activity pattern exhibited by an individual subject and inter-person variability. Therefore these are treated as two individual research problems: event detection and activity recognition. In this paper we concentrate only on the second research problem, activity recognition, on the assumption that the start and stop time of the target activity is known.

In human activity recognition, data collected from inertial sensors is generally analysed using statistical signal processing involving the primary steps of feature extraction, feature selection and classification [16] to monitor simple, gross and dynamic movements, such as sleeping, sitting, standing, cycling, running etc. [1]. By comparison, very little work has been reported in terms of activity recognition for elementary limb movements. A review of the literature shows that different machine learning techniques have been used for movement recognition, e.g. Support Vector Machines (SVM) [22], [25], Decision Trees (DT) [9], [16], Naive Bayes (NB) [16], Multi-Layer Perceptron (MLP) [17], Artificial Neural Networks (ANN) [9], or a combination of these techniques [15]. Hidden Markov Models (HMM) [10] have been used for recognising common gestures made when interacting with objects used in daily living. However, the accuracy of any movement classification technique is dependent on the system components and requirements, covering areas such as: type of activities, number of activities, type of sensors, number of sensors, placement of sensors [23], level of data fusion, etc.

Inertial sensors used for such purposes are susceptible to drift errors, often introduced as result of integration of the raw data, and this error has a quadratic growth over time [26]. To counter this problem, these sensors have been used to complement each other and also used in conjunction with magnetometers to reduce the error [26]-[28]. Complimentary filtering, using quaternion representation [29], Kalman filtering with rotation matrix representation [28] and extended Kalman filtering [30] have been widely used to combine data from inertial sensors and have produced satisfactory results in tracking human body posture [29], [30] and dynamic movements such as walking and running [26], as well as anatomical movements of the back [28].

However, all of these techniques that involve feature extraction and classification or posture tracking through complimentary filtering are computationally intensive and hence may not yield an energy efficient solution that may be applicable in a resource-constrained WBAN node. This therefore motivated us to develop a low-complexity movement 
recognition technique that may be implemented in a WBAN node suitable for long time continuous monitoring applications.

\section{Experimental Protocol}

In this investigation, experiments were performed at the University of Southampton (UoS) with four healthy subjects (age range 24 to 40, male, all right arm dominant) and at the Brandenburg Klinik (BBK) with four stroke impaired patients (age range 45 to 73 , both sexes, both left and right arm dominant). Experiments were performed within an open laboratory with an attached kitchen at UoS and within a treatment centre at BBK under the supervision of the expert physiotherapist members of the research team, though using the same equipment. All involved team members had trained together in the use of the equipment prior to commencing these investigations. Healthy participants were requested to perform the chosen ADL four times within the same session, with a 10 minute rest period between repetitions. By comparison, stroke patients were requested to perform the ADL only twice since they tend to tire quicker and hence performed only whilst they felt comfortable to do so.

\subsection{Movement Selection}

We designed an activity-list that simulated the process of 'making-a-cup-of-tea', as illustrated in Table 1. This is a common activity performed in daily life, which comprises repeated occurrences of three elementary types of arm movement:

- Action A - Reach and retrieve an object (extension and flexion of the forearm).

- Action B - Lift cup to mouth (rotation of the forearm about the elbow).

- Action C-Perform pouring or (un)locking action (rotation of the wrist about long axis of forearm).

\begin{tabular}{|c|c|c|}
\hline \multicolumn{2}{|c|}{ Activity } & \multirow{2}{*}{$\frac{\text { Action }}{\text { A }}$} \\
\hline 1. & Fetch cup from desk & \\
\hline 2. & Place cup on kitchen surface & A \\
\hline 3. & Fetch kettle & A \\
\hline 4. & Pour out extra water from kettle & $\mathrm{C}$ \\
\hline 5. & Put kettle onto charging point & A \\
\hline 6. & Reach out for the power switch on the wall & A \\
\hline 7. & Drink a glass of water while waiting for kettle to boil & $\mathrm{B}$ \\
\hline 8. & Reach out to switch off the kettle & A \\
\hline 9. & Pour hot water from the kettle in to cup & $\mathrm{C}$ \\
\hline 10. & Fetch milk from the shelf & A \\
\hline 11. & Pour milk into cup & $\mathrm{C}$ \\
\hline 12. & Put the bottle of milk back on shelf & A \\
\hline 13. & Fetch cup from kitchen surface & A \\
\hline 14. & Have a sip and taste the drink & $\mathrm{B}$ \\
\hline & Have another sip while walking back to desk & $\mathrm{B}$ \\
\hline 16. & Unlock drawer & $\mathrm{C}$ \\
\hline 17. & Retrieve biscuits from drawer & A \\
\hline 18. & Eat a biscuit & $\mathrm{B}$ \\
\hline 19. & Lock drawer & $\mathrm{C}$ \\
\hline 20. & Have a drink & $\mathrm{B}$ \\
\hline
\end{tabular}

Table1: Use case activity list - 'Making-a-cup-of-tea'

The activity list in our experimental protocol comprises 20 individual activities including 10 occurrences of Action $A, 5$ of Action $B$ and 5 of Action $C$. The start and stop time of the activities were recorded by the researcher observing the subjects as they performed the designated actions during the trial. There were no restrictions on the various physical factors of the experiment such as the seating position, height of the chair or kitchen surface, distance between the chair and the table, standing position with respect to the kitchen surface, position of the objects on the table or kitchen surface 
and the time required to complete the actions. Un-constraining the experiment in this manner helps to generate a wider range of variability in the data which ultimately results in the development of an arm movement recognition system that is more likely to function with acceptable levels of accuracy in a real world application. For the purpose of this conceptual study we do, however, constrain our activity definitions to movements that involve motion of the forearm predominantly within a horizontal plane (the transverse plane, in terms of human anatomy).

\subsection{Sensor Selection and Placement}

The commercially available Shimmer 9DoF wireless kinematic sensor module, consisting of mutually orthogonal tri-axial accelerometers, rate gyroscopes and magnetometers, was used as the sensing platform [31]. For healthy subjects, the dorsal side of the forearm proximal to the wrist on the dominant arm was chosen as the sensing position since it was likely to produce significant sensor responses to the arm movements being investigated. For stroke patients, the same location was selected on the impaired arm since it is the use of this arm in which we are interested with respect to rehabilitation. The Shimmer sensor was fastened to the arm using an elastic strap, providing an intimate and secure, yet unconstraining attachment.

The Shimmer sensors have an internal $2 \mathrm{~Gb}$ data storage capacity (smart card) as well as low power radio communication capabilities (Bluetooth and IEEE 802.15.4) allowing both long-term autonomous data acquisition and real-time monitoring for experimental purposes. Each sensor module weighs $27 \mathrm{~g}$ and measures $53 \mathrm{~mm}$ x $32 \mathrm{~mm}$ x 19 $\mathrm{mm}$, thereby posing minimal obtrusion and discomfort for use over long periods. For our experiments we only use the tri-axial accelerometer with $\pm 1.5 \mathrm{~g}$ range, where $\mathrm{g}$ is the acceleration due to gravity. The magnetometer is excluded in our study since this can be affected by the presence of ferromagnetic materials which are expected to be present in the natural environment (e.g. cooker, wheelchair, etc.) [32]. The gyroscope was also excluded since we aimed to produce a movement detection system using a minimal number of sensors and with a minimal amount of data processing. Furthermore, gyroscopes tend to use more electrical power than accelerometers which would therefore limit their operational lifetime in battery operated WBANs. Sensor data is collected at a rate of $50 \mathrm{~Hz}$, deemed sufficient for assessing habitual limb movement. The sensors transmit kinematic data along with a time stamp to a host computer using the Bluetooth wireless standard and the data corresponding to each activity was segmented using the annotations from the researcher prior to subsequent processing. Since our focus was primarily on recognizing the occurrence of these elementary movements, we did not implement an automated segmentation method. Hence, we refer to this experimental setup as a semi-naturalistic scenario [33].

\subsection{Sensor Calibration}

The accelerometers were calibrated prior to performing any measurements. This can be a very simple process, making use of the omnipresent gravitational acceleration $(\mathrm{g})$ as a reference standard. The accelerometer is placed on a flat surface with its sensing axis either aligned, opposed or orthogonal to the direction of gravitational acceleration, causing it to experience accelerations equal to $+1 \mathrm{~g},-1 \mathrm{~g}$ or $0 \mathrm{~g}$ respectively. Hence a simple 3-point calibration procedure can be implemented without the need of additional equipment, and this simple calibration is adequate if it is known that the accelerometer exhibits a linear response to acceleration. However, three calibration points that define the upper, lower and midrange values of a measurand can be deceptive because a linear or sine function can also be fitted to them with high correlation. It is therefore highly recommended that where practical, calibrations are performed with more than three reference values. For an accelerometer, additional reference accelerations can be experienced by placing it on an inclined surface with known inclination angle so that the sensor axis experiences a component of the gravitational acceleration. With bi-axial and tri-axial accelerometers where the axes are mutually orthogonal, this 
technique has the advantage that a second accelerometer axis simultaneously experiences a different component of the gravitational acceleration based on an angle that is complementary to the slope inclination angle.

To perform a more accurate calibration of the tri-axial accelerometers, we first fabricated plastic wedges with faces inclined at $10^{\circ}, 20^{\circ}, 30^{\circ}$ and $40^{\circ}$ to the horizontal. By placing the Shimmer sensor on each sloped surface and in each of its 24 possible standard orientations (6 faces, any notional edge pointing up, down, left or right), we are able to record the accelerometer responses from each axis over 19 different reference accelerations. The shape of the Shimmer sensor is not ideal, however, for this form of calibration. Its external packaging has tapered sides and certain other features that are slightly in relief on two faces (e.g. nuts, bolts, LEDs), meaning that it cannot be laid down perfectly flat on the sloped surfaces. To counter this, a customised sensor module housing was fabricated with perfectly parallel sides to contain the sensor module during calibration, as illustrated in Fig. 1.

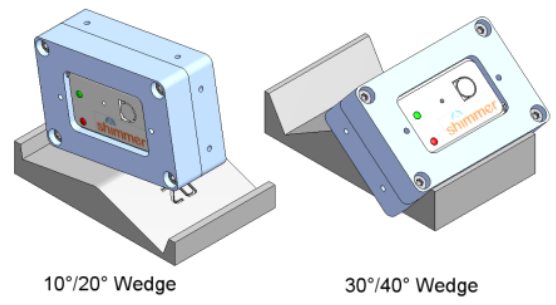

Fig. 1. Illustration of Shimmer 9DoF sensor module enclosed in housing and resting on sloped wedges in different orientations during calibration.

An example of calibration data collected from the Shimmer 9DoF sensor module is shown in Fig. 2, which reveals that the accelerometers have a highly linear response over the acceleration range $\pm 1 \mathrm{~g}$ and quite a large difference in offset values. By applying a least mean square linear fit to the calibration data, individual values for acceleration sensitivity and offset were determined for each accelerometer axis.

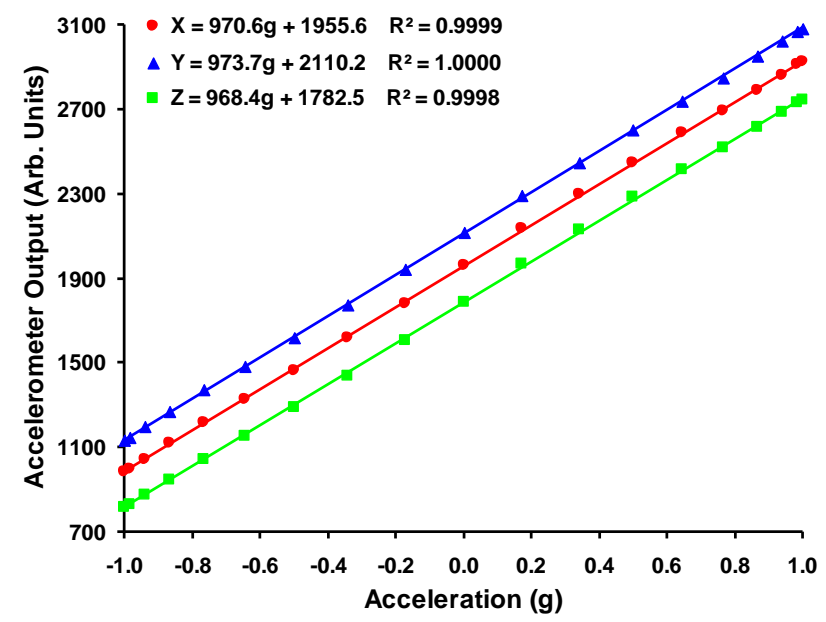

Fig. 2. Example accelerometer calibration data showing highly linear responses over acceleration range $\pm 1 \mathrm{~g}$ with excellent correlation $\left(\mathrm{R}^{2}\right)$ values

\section{Algorithm Design}

The algorithm is based on predicting the most likely orientation of the sensor module at any particular time by assessing which of the three accelerometer axes is the most active at that time. Specific arm movements are then inferred by detecting transitions between sensor module orientations. This is a relatively simple process when the arm is stationary since the total acceleration measured by the sensor module is equal to the value of the gravitational acceleration (g) and its distribution over the three mutually orthogonal accelerometer axes directly indicates the sensor module orientation. 
To appreciate how this simple algorithm works, it is necessary to first understand how a MEMS type accelerometer responds to acceleration. Typically a MEMS accelerometer measures the displacement of a suspended seismic mass either by detecting changes in strain via a piezoresistive element at the root of a cantilever beam to which the seismic mass is attached, or by detecting changes in capacitance of a micro-machined structure where the seismic mass forms one plate of a capacitor. The Shimmer sensor uses the Freescale MMA7361L tri-axial accelerometer which operates using the capacitive principle, as illustrated in Fig. 3. Here, the seismic mass is free to move between two fixed plates, effectively forming two back-to-back capacitors, whose values can be measured using a differential charge amplifier.

All accelerometers respond not only to physical acceleration but also to gravitational acceleration. How an accelerometer acts in response to these two different types of acceleration is quite different. The force of gravity is ubiquitous and is the process which gives mass the quantity of weight. This attractive force is also bi-directional, meaning that any object on the surface of the Earth is attracted toward the centre of the Earth with the same magnitude of force as that object attracts the Earth toward it. Correspondingly, there are two vectors for acceleration due to gravity: one accelerating an object toward the center of the Earth and the other accelerating the Earth toward the centre of the object. However, because the Earth is considerably more massive than most Earth bound objects, it is only the acceleration vector that acts on the object accelerating it in a direction toward the Earth that is observable (cf. $\mathrm{F}=$ ma). Consequently, the seismic mass located within the accelerometer is pulled toward the Earth with an acceleration of magnitude g.

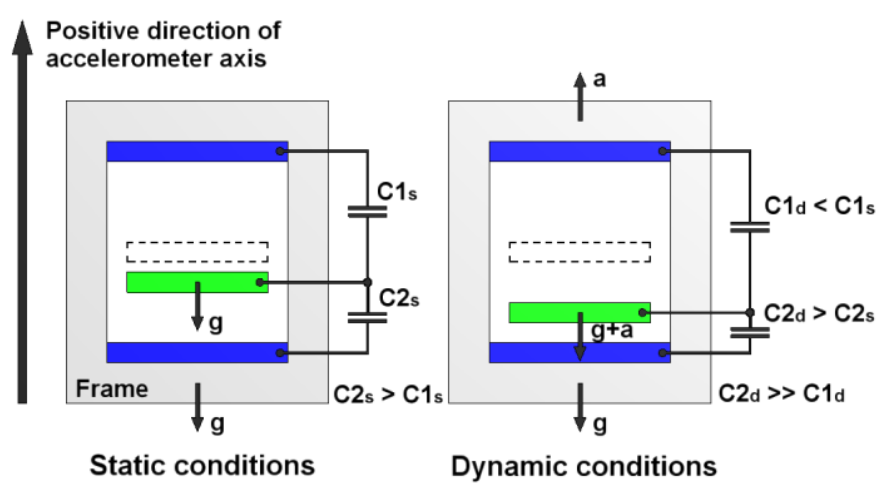

Fig. 3. Operation of a differential capacitive type MEMS accelerometer. The rectangle with broken line shows the initial position of the seismic mass when no acceleration is experienced in the direction of the accelerometer axis.

By comparison, when an accelerometer is physically accelerated (a) by an external force acting on it, the frame of the sensor moves forward in the direction of acceleration but the suspended seismic mass moves backward (due to inertia) with an equivalent acceleration. This will result in the counter-intuitive situation shown in Fig. 3 whereby the seismic mass moves in the same direction when under the influence of gravity alone or when physically accelerated in the opposite direction to that of gravitational acceleration. Put another way, gravitational acceleration acts on all objects and is referenced globally, whereas physical acceleration acts locally and is referenced to the frame of the accelerometer.

With the configuration shown in Fig. 3, the capacitors are connected to processing circuitry such that when $\mathrm{C} 2$ is greater than $\mathrm{C} 1$, the output of the sensor has a positive value. This means that for the orientation shown in the figure, an upward acceleration in the direction of the accelerometer axis results in a positive output. It also means that when stationary, the accelerometer still produces a positive response, but of lower magnitude, despite the fact that gravitational acceleration opposes the direction of the accelerometer axis.

We define 6 reference orientations of the sensor module in the horizontal plane, referred to as Positions 1-6, as illustrated in Fig. 4. In each diagram the dorsal side of the forearm is in contact with the XY plane of the sensor, the X- 
axis points toward the hand and the Z-axis points away from the dorsal aspect. The positions shown cater for all orientations expected when performing the target actions with the sensor module secured to either the right or left arm, and with forearm movement constrained to the horizontal plane. The figure also shows the distribution of the gravitational acceleration between the three accelerometer axes when the forearm is stationary at each position.

With reference to Fig. 4, Positions 1 to 4 represent sequential $90^{\circ}$ rotations of the forearm about the median axis of the arm (i.e. rotation about the $\mathrm{X}$-axis accelerometer), and transitions between subsets of these positions occur with activities such as using a key to open or close a lock or when performing a pouring action (i.e. Action C). The transition from Position 1 to Position 5 represents a $90^{\circ}$ rotation of the forearm about the elbow (i.e. rotation about the Z-axis accelerometer), which occurs when the sensor is worn on the left arm and the forearm is rotated in a drinking action (i.e. Action $B$ ). The corresponding orientation transition for when the sensor is worn on the right arm is given by Position 3 to Position 6.

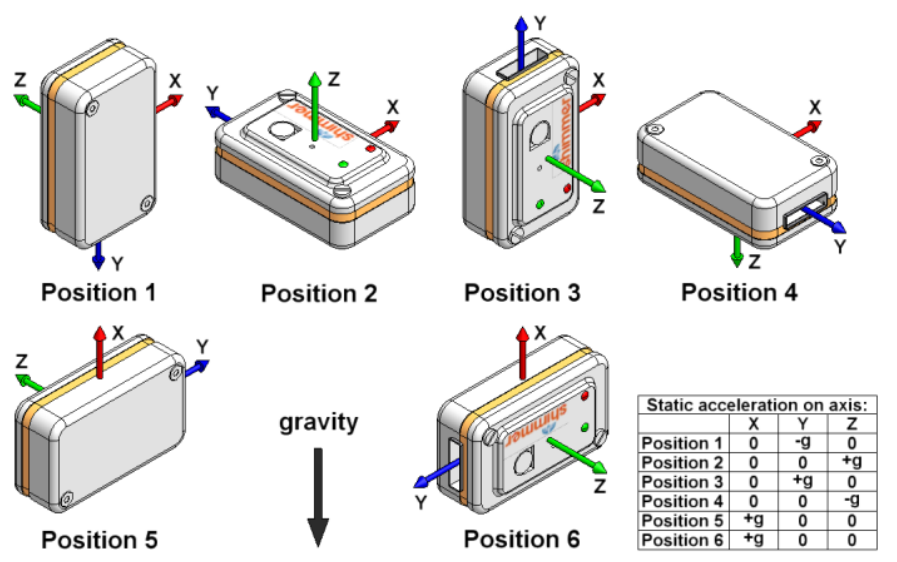

Fig. 4. Predefined orientations of the sensor module with respect to the direction of gravity, showing positive directions of accelerometer axes.

The third arm movement in which we are interested (Action A) is not as simple to detect since it may not necessarily involve a change in orientation of the sensor. Typically, a reach and retrieve action may be performed with the sensor remaining in Position 2 (e.g. hand palmar side down grasping an object such as when using a computer mouse) or remaining in Position 3 when worn on the right arm or Position 1 when worn on the left arm (e.g. hand midway between pronation and supination such as when grasping a mug). The sensor may also remain in Position 4 during a reach and retrieve action, such as when accepting an object in the palm of the hand, though none of the activities defined in our activity list use this type of movement. Quite frequently, Action A involves a change in sensor orientation as the forearm rotates to adopt a particular prehensile shape for the hand prior to grasping an object. Often this means that the orientation of the hand during the retrieve phase of the motion is not the same as that during the reach phase.

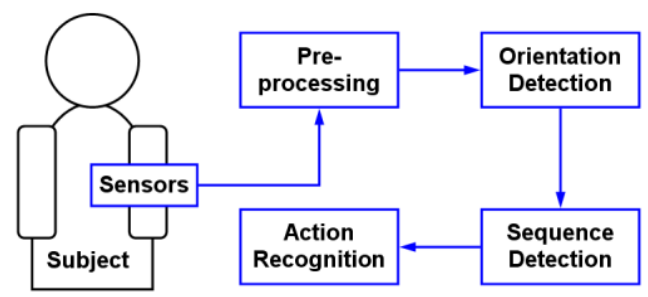

Fig. 5. Basic steps of movement detection using sensor orientation.

The key steps involved in our algorithm, which has been implemented in MATLAB, are illustrated in Fig. 5 and described in the following sections. An overview of the algorithm used to detect and classify each action is also presented in the flow chart shown in Fig. 6. 


\section{1) Pre-processing}

Raw accelerometer data is converted to physical values of acceleration expressed in units of ' $\mathrm{g}$ ' using individual sensor calibration coefficients. The event markers signifying the start and end of each activity that were recorded during data capture are used to segment the data into the 20 activities listed in Table 1. Each data segment is then filtered using a 3rd order low-pass Butterworth filter with a cut-off frequency of $5 \mathrm{~Hz}$ to suppress the high frequency noise components.

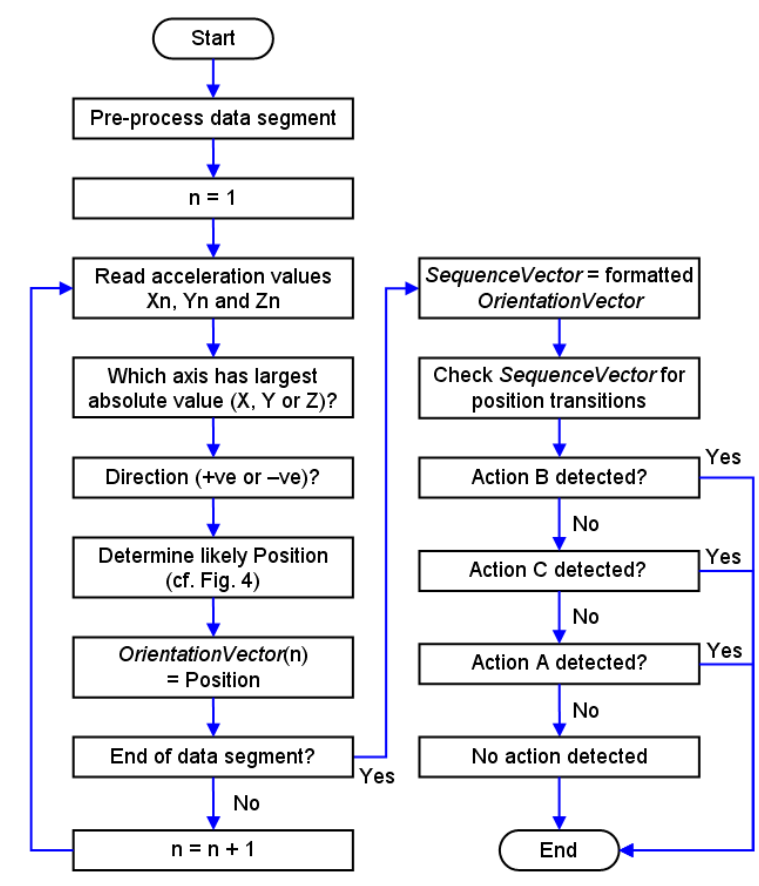

Fig. 6. Flowchart for arm movement detection.

\section{2) Orientation Detection}

Each data segment contains a number of individual data samples, which in turn are comprised of three acceleration values - X, Y and Z. For each data sample we determine the maximum absolute value, its direction, and on which axis this occurred. This information is used to determine the most likely orientation of the sensor module and the identifying number of the corresponding position is stored as an element in an OrientationVector. The rules used to determine the orientation are as follows:

- If the maximum acceleration occurs on the $\mathrm{Y}$-axis is negative, and lies within the range $-\mathrm{g} \pm 0.5 \mathrm{~g}$, we denote the corresponding orientation of the sensor as Position 1.

- If the maximum acceleration occurs on the $\mathrm{Z}$-axis is positive, and lies within the range $\mathrm{g} \pm 0.5 \mathrm{~g}$, we denote the corresponding orientation of the sensor as Position 2.

- If the maximum acceleration occurs on the $\mathrm{Y}$-axis is positive, and lies within the range $\mathrm{g} \pm 0.5 \mathrm{~g}$, we denote the corresponding orientation of the sensor as Position 3.

- If the maximum acceleration occurs on the $\mathrm{Z}$-axis is negative, and lies within the range $-\mathrm{g} \pm 0.5 \mathrm{~g}$, we denote the corresponding orientation of the sensor as Position 4.

- If the maximum acceleration occurs on the $\mathrm{X}$-axis, is positive, and lies within the range $\mathrm{g} \pm 0.5 \mathrm{~g}$, we denote the corresponding orientation of the sensor as Position 5 if worn on the left arm or Position 6 if worn on the right.

- If none of these conditions are met, we assign a value of 0 to the OrientationVector indicating an unknown position.

As described above, the decision as to which orientation the sensor module lies is based on acceptable acceleration 
ranges to allow for the fact that subjects may not orientate their arm such that the sensor module is exactly in one of the orthogonal positions shown in Fig. 4. In practice, the forearm may be slightly rotated and the sensor module will therefore be tilted with respect to the pre-defined positions. Furthermore, basing orientation determination on a range of acceleration values caters for stroke patients who exhibit varying degrees of tremor in the upper limb depending on their time post-stroke. Limit values for these ranges of $\pm 0.5 \mathrm{~g}$ were established experimentally and gave the best action detection rates.

\section{3) Sequence Detection}

Having established the most probable orientation of the sensor module for each set of acceleration data recorded, a SequenceVector is constructed from the annotated positions stored in the OrientationVector, wherein a sequence is defined as a continuous set of orientations of the same type and spanning a minimum duration. This process involves initially passing the OrientationVector through a median filter to remove all zeroes (unidentified orientations). The remaining set of position numbers is further scanned and any repeating sequence with a length less than 13 samples (representing 0.26 seconds of data) is discarded. This action is performed since we consider that the forearm is only truly orientated in a particular position if it remains in that position for at least one quarter of a second. The remaining set(s) of position identifiers forms the SequenceVector.

\begin{tabular}{|c|c|c|c|c|}
\hline \multicolumn{3}{|c|}{ Position Transitions } & Arm & Action \\
\hline \multicolumn{3}{|c|}{ Remaining in Positions 1,2 or 3} & Both & A \\
\hline $1 \longrightarrow 2$ & or & $2 \rightarrow 1$ & Left & A \\
\hline $1 \rightarrow 2 \rightarrow 1$ & or & $2 \rightarrow 1 \rightarrow 2$ & Left & A \\
\hline $3 \rightarrow 2$ & or & $2 \rightarrow 3$ & Right & A \\
\hline $3 \longrightarrow 2 \longrightarrow 3$ & or & $2 \rightarrow 3 \rightarrow 2$ & Right & A \\
\hline \multicolumn{3}{|l|}{$1 \rightarrow 5 \rightarrow 1$} & Left & B \\
\hline \multicolumn{3}{|c|}{$3 \rightarrow 6 \rightarrow 3$} & Right & B \\
\hline \multicolumn{3}{|c|}{ Any transition between subsets of Positions 1 to 4} & Both & $\mathrm{C}$ \\
\hline
\end{tabular}

Table 2: Sequence transitions and corresponding actions. Action A transitions involve additional processing to distinguish them from C.

\section{4) Action Recognition}

The SequenceVector is examined to detect pre-defined transitions between identified positions that correspond to the target actions, as described in Table 2. Firstly, we analyse the SequenceVector to check for Action B, which is a simple transition from Position 1 to Position 5 then back to Position 1 when the sensor is worn on the left arm or from Position 3 to Position 6 and back to Position 3 when the sensor is worn on the right arm. This movement is indicative of a drinking activity, as depicted in Fig 7.

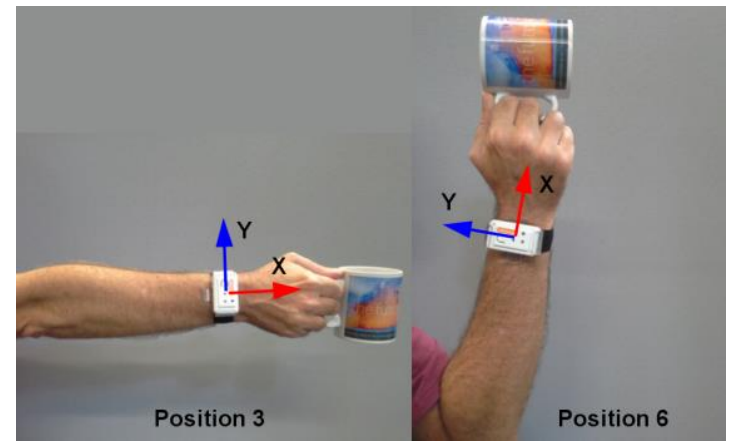

Fig. 7. Transition from Position 3 to Position 6, corresponding to a drinking type of activity (Action B) when sensor worn on right arm.

Secondly, we search the SequenceVector for those orientation transitions that are indicative of Action $C$, which involves rotation of the arm about the median axis. This action may involve a number of different transitions between 
Positions 1, 2 and 3. For example, a pouring action is described by the position sequence 3 to 2 to 1 (or just 3 to 2 ) for when the sensor is worn on the right arm, and by the sequence 1 to 2 to 3 (or just 1 to 2 ) when the sensor is worn on the left arm. For both left and right arm worn sensors, we notice that Position 4 is used when extra torque is required in the rotation action, for example when using a key and lock.

Once a decision about Action $B$ and Action $C$ have been made, we proceed to recognise the occurrence of Action A (reach and retrieve), which is a very generic activity performed in daily life. Action A can result in several different transitions in our activity list, or demonstrate no transitions at all (cf. Table 2). When we reach out for an object in the forward direction, the sensor may remain in Position 1, Position 2 or Position 3 throughout the duration of the activity or it may change between combinations of these Positions depending on the starting position of the forearm and the type of object to be retrieved (i.e. how it will be grasped in the hand). Hence the occurrence of Action A has to be confirmed by additional processing.

To identify Action A, the SequenceVector is first examined to determine how many sequences of consecutively identified orientations it contains. This indicates the number of state changes that occurred during the movement. For each individual sequence in the SequenceVector, we discard the accelerometer axis that is aligned with the direction of gravity for that particular orientation (as defined in Fig. 4) and calculate the acceleration ranges (maximum value minimum value) recorded by each of the other two axes over the duration of that sequence. Specifically:

- For Positions 1 and 3, we compute the acceleration ranges for the $\mathrm{X}$ and Z-axes, neglecting the Y-axis.

- For Position 2 we compute the acceleration ranges for the $\mathrm{X}$ and $\mathrm{Y}$-axes, neglecting the Z-axis.

Therefore, any non-zero value calculated for the range from either of these accelerometer pairs would indicate:

- Movement of the forearm within the plane defined by the two accelerometer axes; or

- Rotation of the forearm about either or both of the accelerometer axes.

However, forearm rotation will have already been detected earlier in the algorithm when testing for the occurrence of Action $C$, and therefore the second scenario never arises.

For each orientation sequence, we compare the computed acceleration ranges against an acceptance range of $\pm 0.2 \mathrm{~g}$. For a movement to be recognised as Action A, either or both of the computed acceleration ranges must be larger than this acceptance range (indicating movement within the horizontal plane) for the majority of sequences stored in the SequenceVector, otherwise the movement is considered as an Unknown Action. Limits for this acceptance range of \pm 0.2 $\mathrm{g}$ were determined experimentally and allow for the fact that stroke impaired patients will demonstrate some degree of tremor in their arm movements which could be erroneously interpreted as directional movement.

\section{Experimental Results}

From the experiments performed, with healthy subjects at UoS and stroke impaired patients at BBK, we gathered a wealth of data from the tri-axial accelerometer placed on the dominant wrist while the subjects performed the designated activities. Typical accelerometer data is shown in Fig. 8 whilst results from the analysis are presented in the following sections.

For the purpose of illustration, Fig. 8 shows typical variations in accelerometer data recorded during a single example of each activity for a healthy subject as well as for a stroke patient. It is clear from the figure that the movement profiles and the distribution of data between the three separate accelerometer axes are sufficiently dissimilar for each type of activity to enable a pattern recognition system with a realistic chance of success, and that this is true for both the healthy subject and the stroke patient. Furthermore, it is also clear that the stroke patient took longer to 
complete each activity and with less smoothness of movement. This is beneficial since it provides a larger variability in the data to test the robustness of the methodology.
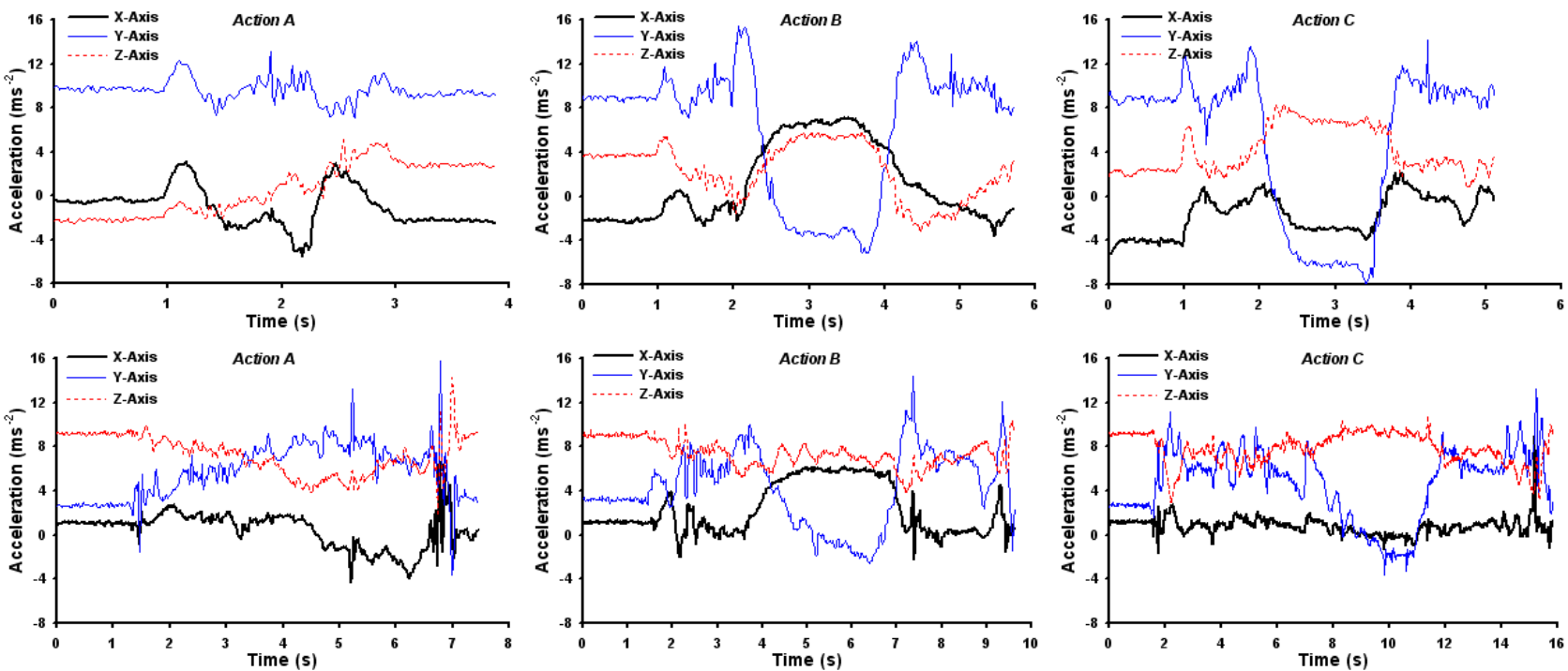

Fig. 8. Example data from a wrist mounted tri-axial accelerometer collected when performing arm Actions $A, B$ and $C$ from a healthy subject (upper) and a stroke patient (lower).

\subsection{Healthy Subjects}

Table 3 presents the results for four healthy subjects each performing 4 trials of the activity-list, each of which involves a total of 20 occurrences of the target actions. We observe that the average accuracy of correctly recognising the 3 actions over the 4 trials for all subjects is within a range of $91 \%-99 \%$.

\begin{tabular}{|c|c|c|c|c|c|}
\hline \multirow{2}{*}{ Subject } & \multicolumn{4}{|c|}{ Recognised Actions (Out of 20) } & \multirow{2}{*}{$\begin{array}{c}\text { Average } \\
\text { Accuracy }(\%)\end{array}$} \\
\hline & Trial 1 & Trial 2 & Trial 3 & Trial 4 & \\
\hline 1 & 19 & 20 & 19 & 20 & 98 \\
\hline 2 & 16 & 20 & 18 & 19 & 91 \\
\hline 3 & 19 & 18 & 19 & 18 & 93 \\
\hline 4 & 20 & 19 & 20 & 20 & 99 \\
\hline
\end{tabular}

Table 3: Recognition of trials for healthy subjects.

\subsection{Stroke Patients}

Table 4 presents the corresponding results for four stroke patients each performing two trials of the designated activity-list. The average recognition accuracy over the two trials for all four patients is within a range of $70 \%-85 \%$.

\begin{tabular}{cccc}
\hline \hline \multirow{2}{*}{ Subject } & \multicolumn{2}{c}{ Recognised Actions (Out of 20) } & $\begin{array}{c}\text { Average } \\
\text { Accuracy (\%) }\end{array}$ \\
\cline { 2 - 4 } & Trial 1 & Trial 2 & 85 \\
2 & 17 & 17 & 70 \\
3 & 14 & 14 & 75 \\
4 & 16 & 14 & 70 \\
\hline \hline
\end{tabular}

Table 4: Recognition of trials for stroke patients.

\section{Discussion and Future Work}

In this paper we have presented a novel yet simple algorithm to recognise three elementary upper limb movements involved in performing a representative activity of daily living in an unconstrained environment, namely the process of 'making-a-cup-of-tea'. The subject wears a sensor module strapped onto the forearm close to the wrist, comprised of a 
tri-axial accelerometer. We use a simple technique, whereby we attempt to recognise the occurrences of 6 pre-defined orientations of the sensor module by examining the distribution of recorded accelerations across the 3 mutually orthogonal accelerometer axes. We analyse the sequence of allocated positions and their transitions to recognise the arm movements performed.

Our results prove the robustness of our algorithm since we apply it on movement data from healthy subjects as well as stroke patients who can have a significantly different movement profile. The fact that we can achieve an accuracy range of $91 \%-99 \%$ for healthy subjects and $70 \%-85 \%$ for stroke patients using only a tri-axial accelerometer sensor on the wrist, demonstrates an alternative approach that reduces the overheads associated with complex data processing algorithms involved in conventional methodologies of human movement recognition. The seemingly low accuracy rates reported for the stroke patients are acceptable, according to clinicians, since it provides a gross measure of impaired arm use. The approach described here has the advantage of negating the requirement of training a system to learn response patterns, as is the case in most conventional pattern recognition systems. The achieved accuracies can be considered favourable given the recognition was performed on activities performed in an out-of-laboratory, semi-naturalistic scenario [34]. The proposed algorithm has low computational complexity, mostly involving comparisons, additions, subtractions and few multiplications. It is therefore suitable for translation into hardware in the form of an ASIC or FPGA, and can therefore be used as a low power processing component in a WBAN activity recognition system aimed at real-time operations. Moreover, in view of its real-time operability, we do not use the power hungry gyroscope, thereby also avoiding the additional amount of data processing related to it.

The algorithm has been developed simply as a proof of concept vehicle for this activity recognition methodology in stroke patients. Results have been encouraging and show that these particular arm movements can be reliably detected with stroke patients exhibiting moderate levels of involuntary tremor in their movements. The algorithm could be extended for use with patients suffering from other neurodegenerative disorders that might demonstrate greater levels of tremor or less fluidic movement profiles. In such circumstances, additional band-pass filtering can be applied to sensor recordings to suppress higher frequency data associated with rapid or discontinuous movements. Hence, a resource constrained remote monitoring system employing this activity recognition methodology would be capable of detecting and recording the various occurrences of prescribed exercises (i.e. arm movements) during daily activities, which over time could provide a measure of rehabilitation progress.

Future work will therefore focus on developing simple algorithms that can detect an extended range of reference orientations that cater for arm movements outside of this plane. This will allow the detection and recognition of a greater range of arm movements, provided that these additional movements can be characterised by other distinct transitions between the extended range of reference orientations. Future work will also concentrate on developing an auto-segmenting algorithm to automatically select, and pass on to the recognition algorithm, that data which potentially contains arm movement information as opposed to processing all acquired data. This is a necessary component of a truly autonomous activity recognition system, particularly one designed to detect and monitor the occurrences of particular body movements during home based rehabilitation. Such an algorithm might be implemented by simply determining the modulus $(\mathrm{M})$ of the combined accelerometer axes (which is a measure of general activity and is given by $M=\sqrt{X^{2}+Y^{2}+Z^{2}}$ ) and comparing this to a preset threshold value.

\section{Acknowledgments}

We would like to thank all participants for their kind cooperation in these experiments. This work was supported by the Information and Communication Technologies Theme of the European Union Seventh Framework Programme, 
under the project name "StrokeBack: Telemedicine system empowering stroke patients to fight back" (grant number 288692).

\section{References}

[1] B. Najafi, K. Aminian, A. Paraschiv-Ionescu, F. Loew, C. J. Bula, and P. Robert, "Ambulatory system for human motion analysis using a kinematic sensor: Monitoring of daily physical activity in the elderly,” IEEE Trans. Biomed. Eng., vol. 50, no. 6, pp. 711-723, Jun. 2003.

[2] A. Hadjidj, A. Bouabdallah, and Y. Challal, "Rehabilitation supervision using wireless sensor networks," in Proc. IEEE WoWMoM, Luca, Italy, 2011, pp.1-3.

[3] K. Maharatna, E. B. Mazomenos, J. Morgan, and S. Bonfiglio, "Towards the development of next-generation remote healthcare system: Some practical considerations," in Proc. IEEE ISCAS, Seoul, Korea, pp. 1-4, 2012.

[4] M. S. Raisinghania, A. Benoit and J. Ding, “Ambient intelligence: Changing forms of human-computer interaction and their social implications," Texas Digital Library, vol. 5, no. 4, 2004.

[5] D. Merrill, J. Kalanithi and P. Maes, "Siftables: Towards sensor network user interfaces," In Proceedings of the 1st international conference on Tangible and Embedded Interaction, Baton Rouge, LA, 2007, pp. 75-78.

[6] S. Armstrong, "Wireless connectivity for health and sports monitoring: a review," B. J. Sports Medicine, vol. 41, pp. $285-289$, Jan. 2007.

[7] M. Ermes, J. Parkka, J. Mantyjarvi, and I. Korhonen, "Detection of daily activities and sports with wearable sensors in controlled and uncontrolled conditions,” IEEE Trans. Inf. Technol. Biomed., vol. 12, no. 1, pp. 20-26, Jan. 2008

[8] R. Zhu and Z. Zhou, “A real-time articulated human motion tracking using tri-axis inertial/magnetic sensors package,” IEEE Trans. Neural Syst. Rehabil. Eng., vol. 12, no. 2, pp. 295-302, Jun. 2004.

[9] J. Parkka, M. Ermes, P. Korpipaa, J. Mantyjarvi, J. Peltola, and I. Korhonen, “Activity classification using realistic data from wearable sensors," IEEE Trans. Inf. Techol. Biomed., vol. 10, no. 1, pp. 119-128, Jan. 2006.

[10] H. Junker, O. Amft, P. Lukowicz, and G. Tröster, "Gesture spotting with body-worn inertial sensors to detect user activities," Pattern Recognition, vol. 41, no. 6, pp. 2010-2024, Jun. 2008.

[11] Y. J. Hong, I. J. Kim, S. C. Ahn, and H. G. Kim, "Mobile health monitoring system based on activity recognition using accelerometer," Simulation Modelling Practice and Theory, vol. 18, no. 4, pp. 446-455, Apr. 2010.

[12] D. T-P. Fong and Y-Y. Chan, "The use of wearable inertial motion sensors in human lower limb biomechanics studies: A systematic review," Sensors, vol. 10, no. 12, pp. 11556-11565, Dec. 2010.

[13] G. X. Lee, K. S. Low, and T. Taher, "Unrestrained measurement of arm motion based on a wearable wireless sensor network," IEEE Trans. Instrum. Meas., vol. 59, no. 5, pp. 1309-1317, May 2010.

[14] Y. Jung, D. Kang, and J. Kim, “Upper body motion tracking with inertial sensors,” in Proc. IEEE ROBIO, Tianjin, China, 2010, pp. $1746-1751$.

[15] C. Zhu and W. Sheng, "Motion- and location-based online human daily activity recognition," Pervasive and Mobile Computing, vol. 7, no. 2, pp. 256-269, Apr. 2011.

[16] O. Banos, M. Damas, H. Pomares, A. Prieto and I. Rojas, "Daily living activity recognition based on statistical feature quality group selection," Exp. Syst. Appl., vol. 39, no. 9, pp. 8013-8021, July 2012.

[17] S. Chernbumroong, S. Cang, A. Atkins, and H. Yu, "Elderly activities recognition and classification for applications in assisted living," Exp. Syst. Appl., vol. 40, no. 5, pp. 1662-1674, Apr. 2013.

[18] S. Patel, R. Hughes, T. Hester, J. Stein, M. Akay, J. G. Dy, and P. Bonato, "A novel approach to monitor rehabilitation outcomes in stroke survivors using wearable technology," Proc. IEEE, vol. 98, no. 3, pp. 450-461, Mar. 2010.

[19] S. K. Subramanian, J.Yamanaka, G. Chilingaryan, M. F. Levin, "Validity of movement pattern kinematics as measures of arm motor impairment poststroke," Stroke, vol. 41, pp. 2303-2308, Jul. 2010.

[20] E. Jovanov, A, Milenkovic, C, Otto, and P. C. de Groen, “A wireless body area network of intelligent motion sensors for computer assisted physical rehabilitation," J. NeuroEng. Rehabil., vol. 2, no. 6, Mar. 2005.

[21] P. Bonato, “Advances in wearable technology and applications in physical medicine and rehabilitation,” J. NeuroEng. Rehabil., vol. 2, no.2, Feb. 2005.

[22] A. Fleury, M. Vacher, and N. Noury, "SVM-based multimodal classification of activities of daily living in health smart homes: Sensors, algorithms, and first experimental results,” IEEE Trans. Inf. Technol. Biomed., vol.14, pp. 274-283, Mar. 2010.

[23] D. Fuentes, L. Gonzalez-Abril, C. Angulo, and J. A. Ortega, "Online motion recognition using an accelerometer in a mobile device," Expert Systems with Applications, vol. 39, no. 3, pp. 2461-2465, Feb. 2012.

[24] F. E. Martınez-Pérez, J. A. González-Fraga, J. C. Cuevas-Tello, and M. D. Rodrıguez, "Activity inference for ambient intelligence through handling artifacts in a healthcare environment," Sensors, vol. 12, no. 1, pp. 1072-1099, Jan. 2012. 
[25] G. D. Fulk, and E.Sazonov, "Using sensors to measure activity in people with stroke," Top Stroke Rehabil., vol. 18, no. 6, pp. 746-757, Nov. 2011

[26] X. Yun, E.R. Bachmann, H. Moore IV, and J. Calusdian, "Self-contained position tracking of human movement using small inertial/magnetic sensor modules," in IEEE Int. Conf. Robotics Auto., Rome, Italy, 2007, pp. 2526-2533.

[27] H. Zhou and H. Hu, "Human motion tracking for rehabilitation-A survey," Biomed. Sig. Process. Control, vol. 3, no. 1, pp. 1-18, Jan. 2008.

[28] D. Roetenberg, P.J. Slycke, and P.H. Veltink, “Ambulatory position and orientation tracking fusing magnetic and inertial sensing," IEEE Trans. Biomed. Eng., vol. 54, no. 5, pp. 883-890, May 2007.

[29] E. R. Bachmann, X. Yun, and R.B. McGhee, "Sourceless tracking of human posture using small inertial/magnetic sensors." in Proc. IEEE Int. Symp. Comput. Intell. Robo. Auto., vol. 3, pp. 822-829, 2003.

[30] J. L. Marins, X. Yun, E. R. Bachmann, R. B. McGhee, and M. J. Zyda, “An extended Kalman filter for quaternion-based orientation estimation using MARG sensors," in Proc. IEEE/RSJ, vol. 4, Hawaii, USA, pp. 2003-2011, 2001.

[31] A. Burns, B. R. Greene, M. J. McGrath, T. J. O'Shea, B. Kuris, S. M. Ayer, F. Stroiescu, and V. Cionca, "Shimmer - A wireless sensor platform for noninvasive biomedical research," IEEE Sensors Journal, vol. 10, no. 9, pp.1527-1534, Sep. 2010.

[32] C. Kendell and E. D. Lemaire, "Effect of mobility devices on orientation sensors that contain magnetometers," J. Rehab. Res. Dev., vol. 46, no.7, pp. 957-962, 2009

[33] L. Bao and S. Intille, "Activity recognition from user-annotated acceleration data", in Proc. $2^{\text {nd }}$ Int. Conf. Pervasive Computing, vol. 3001, pp. 1-17, 2004.

[34] M. Ermes, J. Parkka, J. Mantyjarvi, and I. Korhonen, "Detection of daily activities and sports with wearable sensors in controlled and uncontrolled conditions," IEEE Trans. Inf. Technol. Biomed., vol. 12, no.1, pp. 20-26, Jan. 2008. 